\title{
The influence of the secondary geodynamic factors on medico-biological and economic risks
}

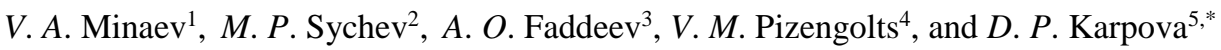 \\ ${ }^{1}$ Dr. of Technical Sciences, Professor Sciences, Professor of Bauman State technical University, \\ ul. Baumanskaya 2-ya, 5, 105005, Moscow, Russian Federation \\ ${ }^{2}$ Dr. of Technical Sciences, Professor of Ryazan State University named for S. Yesenin, \\ ul. Svobody 46, 390000, Ryazan, Russian Federation \\ ${ }^{3}$ Dr. of Technical Sciences, Professor, Head of the regional training and research centre "Security" of \\ Bauman State technical University, ul. Baumanskaya 2-ya, 5, 105005, Moscow, Russian Federation \\ ${ }^{4}$ Dr. Of Economical Sciences, Professor of the Peoples ' Friendship University of Russia (RUDN \\ University), 6 Miklukho-Maklaya St., Moscow, 117198, Russian Federation \\ ${ }^{5} \mathrm{PhD}$, Associate Professor of the Peoples ' Friendship University of Russia (RUDN University), \\ 6 Miklukho-Maklaya St., Moscow, 117198, Russian Federation
}

\begin{abstract}
Influence of secondary geodynamic factors on medical and biological safety of population is considered. Among these factors are electromagnetic and infrasound fields caused by "slow" geodynamic nature catastrophes discussed. Some features and causes of "slow catastrophes", as well as the specifics of infrasonic oscillations are described. The hypothesis about the influence of infrasound field on the incidence of cancer diseases considered. The possibility of application of the proposed research methods in the planning of development and operation of modern settlements.
\end{abstract}

\section{Dangers of secondary geodynamic factors}

The results of numerous studies convincingly prove the existence of an indissoluble connection of processes and phenomena occurring in various earthly "spheres" [1-6]. Anomalous phenomena in the geophysical environment have a multi-level character and are manifested as complex, reflected in the form of earthquakes in the lithosphere, unusual and especially dangerous phenomena, cyclones in the atmosphere, magnetic storms in the magnetosphere and ionospheric disturbances. It is important to note that an abnormal phenomenon occurring in one sphere may cause disturbance in other spheres $[2,7,8]$.

Thus, the change in the total gravitational tension in the vicinity of the Earth is reflected on the planet in the fact that there is an alternate compression and stretching of the earth's crust, causing the accumulation of elastic stresses in the layers of the rock. At the same

* Corresponding author: karpova_dp@rudn.ru 
time, when tectonic blocks, plates are engaged, the lithospheric electromagnetic fields in the compression region will be suppressed, and in the area of stretching they will increase. In the atmosphere, the directed motion of charged particles will begin, causing the generation of powerful electromagnetic fields [9-11].

As is known, the electric field intensity in the surface layer under normal conditions varies between 100 and $200 \mathrm{~V} / \mathrm{m}$ [12]. And in an earthquake, the electric field strength near the earth's surface increases a thousand times, up to $200,000 \mathrm{~V} / \mathrm{m}$. So, in the Carpathian earthquake of 1986 with a magnitude of about 7 a day before the earthquake at a distance of 200-250 km, in Bucharest, almost all computers failed.

The influence of such a powerful electric field on humans and electronic technology is called a geodynamic factor. His study is devoted to a number of works [13-15].

The dependence of the concentration of charged particles on the magnitude of the electrostatic field is interesting (Fig.(1 ) that is, [14]. Starting with a certain value of the concentration of mobile carriers, an avalanche-like increase in the concentration of ions occurs, and an independent discharge begins in the atmosphere (site No. 3). It is at this stage and there is a glow of the atmosphere in the sky visible flashes and lightning.

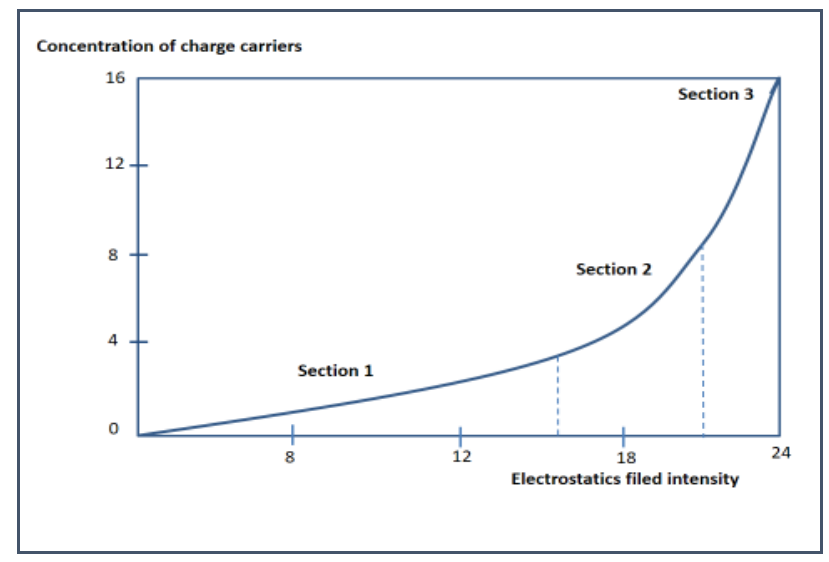

Fig. 1. Dependence of the concentration of mobile charge carriers in atmospheric air (in conventional units) on the electrostatic field strength (in conventional units).

In addition to the electric field, in catastrophic earthquakes (8 points or more) there are many more dangerous consequences - all sorts of injuries that occur due to the collapses and collapses of walls and roofs, from the fall of parts of buildings and unjustified actions of people in a panic and fear. There are statistical data on the behaviour of people in extreme conditions of this kind in areas of high intensity (see figure 2, on the example of the Tashkent earthquake in 1966). Attention is drawn to the fact of a clear excess of the number of injuries caused by the mental state of people (55\%) over others combined [13]. Far from the epicenter 3-4 point zone manifestations of a strong earthquake at frequencies of $0.3-0.5 \mathrm{~Hz}$ and the amplitude of the displacement of the soil $0.1-0.5 \mathrm{~mm}$, some people, especially women, feel dizzy. In the 4-5-point zone, where the displacement amplitudes are $1.0-4.0 \mathrm{~mm}$, in the same frequency band quite a significant number of people experience a state of nausea. With concussions of 7-8 points, mainly in the epicentral zone, the highfrequency spectrum of oscillations $1.0-30 \mathrm{~Hz}$ has a vibration amplitude of about 4-8 mm. People experience panic fear, lose self-control, etc. $[13,16]$. All this suggests that a person in earthquakes are affected by powerful fluctuations in the range of infrasonic frequencies. 
This is confirmed by studies in the aviation and space industries, at enterprises where there are vibration installations, mechanized works in agriculture, at other facilities [17, 31].

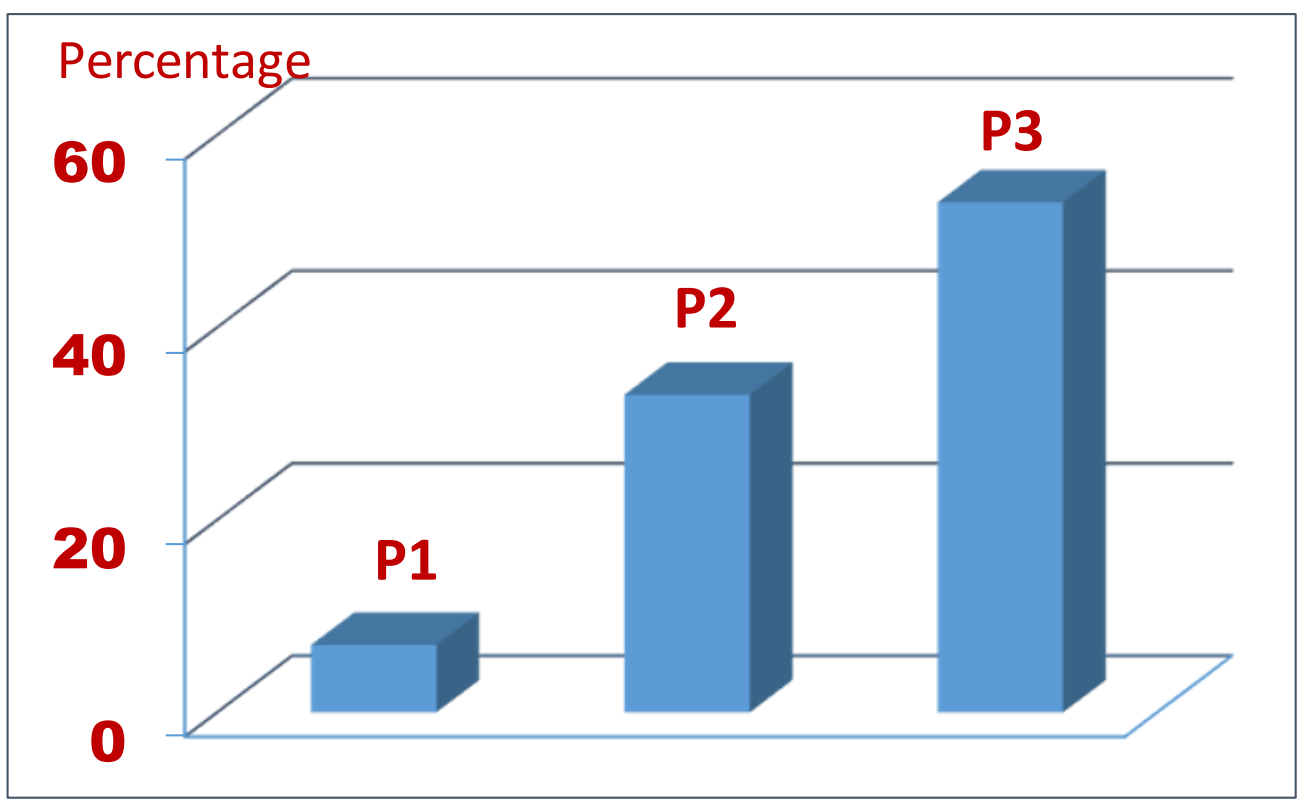

Fig.2. Distribution of the number of injuries and deaths from various causes (in \%): P1- collapses and collapses of walls and roofs; P2 - falling plaster, bricks, household items; P3 - the behaviour of the victims (panic, fear, unconscious actions, jumping out of the windows of the upper floors).

The most dangerous to human health resonant frequencies at which the frequency of natural oscillations of the body or individual organs coincides with the frequency of forced oscillations. At the same time, not only the physiological reactions of the body are enhanced, but also mechanical damage to tissues and organs may occur [17].

The first resonance of the whole body in vibration along the longitudinal axis of the person corresponds to the frequencies of 4-5 Hz, the second occurs at frequencies of $12-24 \mathrm{~Hz}$, at frequencies of $20-30 \mathrm{~Hz}$ there is a resonance of the head, and at $60 \mathrm{~Hz}-$ eyeballs. Low-frequency oscillations (from the parts of Hertz to $3-4 \mathrm{~Hz}$ ) - stimuli of the vestibular apparatus, under the influence of which there are phenomena characteristic of motion diseases (dizziness, nausea, vomiting, sweating, loss of spatial orientation). With mechanical oscillations of the brain inside the cranial box at large amplitudes (more than $4 \mathrm{~mm}$ ) with a frequency of about $7 \mathrm{~Hz}$, people have fear, acute mental diseases occur [17].

And what happens in the case when externally seismic events are not manifested, and there are constant minor geodynamic movements, the so-called "creep"?

In [18-22, 31] the authors studied such types of geodynamic hazards as "slow catastrophes". They are understood as the processes of occurrence of negative trends in the functioning of territorial objects, their subsequent accumulation, development and unpredictable modification, resulting in disruption of their normal functioning, disorganization of the internal structure, disruption of relations with the environment and other negative consequences up to complete destruction.

The sources of preparation and flow of "slow" catastrophes are geodynamic shifts in tectonic faults and accompanying deformation of the upper parts of the earth's crust. Such 
movements lead not only to the appearance and accumulation of deformations in the near surface soils, supports, bearing structures, buildings, structures [5, 23, 24], the development of landslide processes, but also act as one of the reasons for the generation of infrasonic fields of the bioactive range, negatively affecting the bio-and psychophysical processes $[2$, $8,11,24-27]$.

Studies carried out by a number of authors have shown that the health and safety of human life are directly dependent on the fields of bioactive range, genetically related to the network of tectonic fault structures of the upper crust $[2,8,9,13,27,28]$.

The influence of the geodynamic factor-fluctuations in the range of infrasonic frequencies-can be manifested both during the implementation of a major seismic event, and latently, with a gradual accumulation of negative changes in the environment, which is a "slow" disaster in relation to the anthropogenic component of any distributed natural and technical system.

\section{The rating velocities of shear deformations in the geological medium}

This article attempts to assess the risk of medical and biological effects on the population of infrasonic fluctuations that exceed the permissible norms for the territory located in the Central part of the East European platform.

As a model region was taken the real territory of a large city, which investigated the territorial differences of cancer among the population, taking into account their relationship with the spatial distribution of such geophysical characteristics of the geological environment as the modern vertical and horizontal displacement in the geological environment.

Studies have shown that most of the city covered by the process of geodynamic lowering, and it is quite gradient, indicating significant vertical deformation. In the rest of the territory there is a geodynamic uplift, which has a gradient much smaller than the lowering. As for the vectors of horizontal displacements, their orientation suggests that the territory of the city is a rather complex geological structure, "dissected" by a whole set of tectonic faults of the geological environment.

Consider the procedure for estimating the values of shear strains and their velocities in the geological environment of the territory under consideration.

Using data on modern vertical and horizontal displacements, it is possible to calculate the values of shear strains on the basis of the following relations:

$$
\left\{\begin{array}{l}
\gamma_{x z}=\frac{1}{2}\left(\frac{\partial u_{x}}{\partial z}+\frac{\partial u_{z}}{\partial x}\right) \\
\gamma_{y z}=\frac{1}{2}\left(\frac{\partial u_{y}}{\partial z}+\frac{\partial u_{z}}{\partial y}\right) \\
\gamma_{x y}=\frac{1}{2}\left(\frac{\partial u_{x}}{\partial y}+\frac{\partial u_{y}}{\partial x}\right)
\end{array}\right.
$$

where $\gamma_{\mathrm{xz}}, \gamma_{\mathrm{yz}}, \gamma_{\mathrm{xy}}$ shear deformations in the XZ, YZ, XY planes, respectively; $u_{\mathrm{x}}, u_{\mathrm{y}}, u_{\mathrm{z}}-$ components of the full displacement vector in the geological environment. 
That is, according to the relations (1), it is necessary to numerically estimate the gradients of the components of the full displacement vector in the geological medium in the specified directions (along the $\mathrm{x}, \mathrm{Y}$ and $\mathrm{Z}$ coordinate axes).

However, if the gradients of the components $u_{x}, u_{y}, u_{z}$ on the $X$ and $Y$ axes can be estimated uniquely, the gradients of the components $u_{x}$ and $u_{y}$ on the $Z$ axis cannot be determined, since the nature of the change of these values with depth is unknown. Therefore, according to the works $[14,27]$, we make an assumption: the displacements $u_{\mathrm{x}}$, $\mathrm{u}_{\mathrm{y}}, \mathrm{u}_{\mathrm{z}}$ do not depend on the $\mathrm{z}$ coordinate and remain constant throughout the depth of the geomedium layer.

In this case, formulas (1) are converted to the following relations

$$
\left\{\begin{array}{l}
\gamma_{x z}=\frac{1}{2} \frac{\partial u_{z}}{\partial x}, \\
\gamma_{y z}=\frac{1}{2} \frac{\partial u_{z}}{\partial y}, \\
\gamma_{x y}=\frac{1}{2}\left(\frac{\partial u_{x}}{\partial y}+\frac{\partial u_{y}}{\partial x}\right)
\end{array}\right.
$$

Finding the gradients of the components of the full displacement vector in the geological environment included in the ratio (2) was performed using a known numerical differentiation formula at uniformly spaced nodes [29].

The value of the total shear strain at each design point for the study area was determined using the values of shear strains calculated in this way by the formula:

$$
\gamma=\sqrt{\gamma_{x z}^{2}+\gamma_{y z}^{2}+\gamma_{x y}^{2}} .
$$

Recall that to assess the impact of secondary geodynamic factors need not themselves shear strain, and their speed.

Numerical calculations of the velocity values of shear deformations has shown that in order they make the quantity of $10^{-12}-10^{-11} \mathrm{~s}^{-1}$ which is in good accordance with the estimates for platform territories, is given in [14, 27,30].

The analysis of the spatial distribution in the territory of the settlement of the calculated values of shear strain rates allowed to conclude that in most of the territory dominated by significant in its magnitude shear strain rate (more than $1.0 \times 10^{-11} \mathrm{~s}^{-1}$ ), and in some areas $2.0 \times 10^{-11} \mathrm{~s}^{-1}$ and even more $-3.0 \times 10^{-11} \mathrm{~s}^{-1}$.

It is on these sites that manifestations of such dangerous geodynamic processes as landslides, subsidence, dips are observed, and manifestations of secondary geodynamic factors affecting the health and psyche of the population are recorded.

\section{The results of the evaluation of medical-biological safety of territories under the influence of secondary geodynamic factors}

Let us now consider the human impact of the secondary geodynamic factor - infrasonic oscillations generated by deformations in the geological environment. 
Infrasound is characterized by a huge penetrating power, due to its low absorption. Thus, when propagating in the deep sea and in the atmosphere at ground level infrasonic waves of frequency $10-20 \mathrm{~Hz}$ attenuate at a distance of $1000 \mathrm{~km}$ no more than a few DB (decibels). Therefore, due to low absorption and scattering, infrasound can spread over very long distances.

Infrasound pressure was estimated based on the following ratio:

$$
p=2 \pi v \rho V u,
$$

where $v$ - frequency infrasonic vibrations; $\rho$-is the density of the geological environment; $V$ - the speed of propagation of infrasonic fluctuations in the geological environment; $u$ - amplitude of displacements in the geological environment.

We have constructed equipotential distributions of infrasonic pressure values in octave bands with average geometric frequencies of $2 \mathrm{~Hz}, 4 \mathrm{~Hz}, 8 \mathrm{~Hz}$ and $16 \mathrm{~Hz}$ for the territory of the studied settlement. In their structure, these distributions are identical, their difference is only in the values of infrasound pressure generated by the elementary volume of the geological medium, while the pressure values increase in proportion to the increase in the frequency of infrasound.

The authors have estimated the level of infrasound pressure for the following volumes of the geological environment: $100 \mathrm{~m}^{3}, 1000 \mathrm{~m}^{3}, 10000 \mathrm{~m}^{3}$.

For these volumes, the levels of the infrasonic range are very unsafe for a person in the places of his permanent residence (Fig. 3).

And now let's see what is the correspondence of the constructed equipotential distribution of infrasonic pressure levels (for example, the pressure level from $1000 \mathrm{~m}^{3}$ of the volume of the geological environment at the frequency of infrasonic oscillations of $2 \mathrm{~Hz}$ ) and the distribution of oncological diseases in the city.

Because of the high importance and importance of the results, we are not entitled to provide this information in the form of maps, combined with the plan of the settlement, so we offer to the readers only data on the equipotential distribution of infrasound pressure levels, combined in terms of areas of the largest distribution in the city of cancer (Fig. 4).

The relationship between the distribution of the highest levels of infrasonic pressure (exceeding the maximum permissible limits) and the incidence of cancer is obvious. At the same time, we should not forget about the extremely small absorption and scattering of infrasound and its ability to spread over very long distances.

The primary task, and this is the next stage of our future research, is to assess the degree of interconnection of the given data not only visually, but also quantitatively. The fact is that it is possible to talk about quantitative relationships and relationships only if the distribution of cancer diseases for the entire study area is statistically known. Unfortunately, full information on this territory is not available.

Therefore, so far we only hypothetically note that the manifestations of the secondary geodynamic factor associated with the generation of constant geodynamic movements of the infrasound range oscillations can significantly affect not only the comfort but also the safety of human habitation in normal conditions in the background of geodynamic processes. 


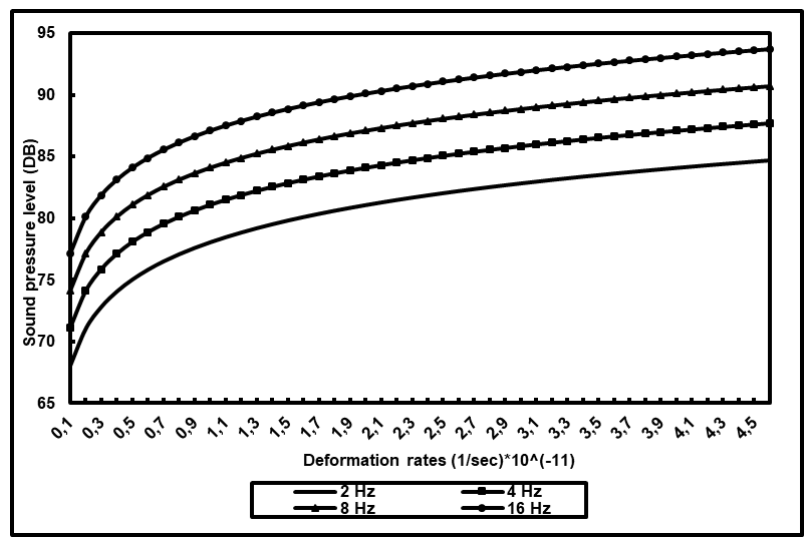

Fig. 3. Dependence of the infrasonic pressure level from $1000 \mathrm{~m}^{3}$ the volume of the geological medium from the values of strain rates in this medium.

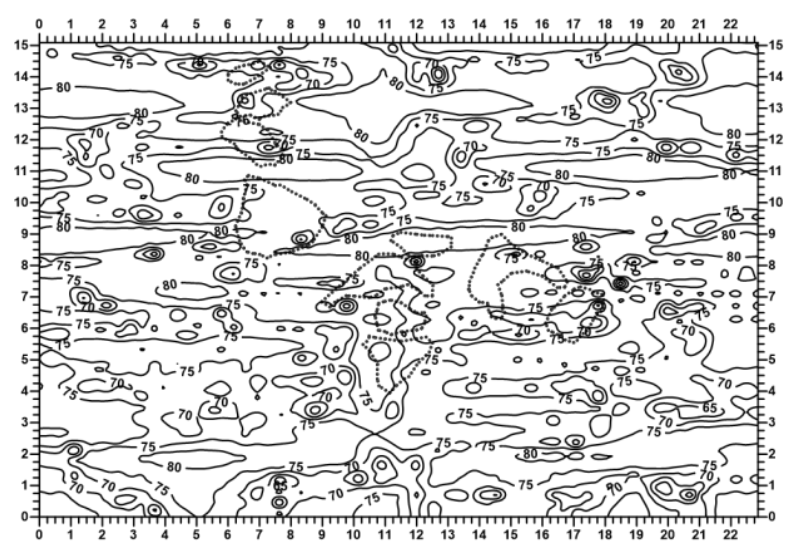

Fig. 4. Equipotential distribution of the infrasonic pressure level, coming from $1000 \mathrm{~m}^{3}$ of geological environment for the territory of the city $\mathrm{N}$ and its vicinities (frequency $2 \mathrm{~Hz}$ ). Areas of the city, unfavorable from the point of view of cancer, are outlined with dotted lines.

\section{Conclusion}

When assessing the safety of populated areas, it is imperative to take into account secondary geodynamic factors in the form of a powerful electric field that affects humans and electronics, and more importantly - fluctuations in the range of infrasonic frequencies that can create medical and biological anomalies for the population, including an increased level of cancer.

This publication has been prepared with the support of the "RUDN University Program 5-100".

\section{References}

1. S. K. Shoigu, GKChS (1992)

2. Atlas of temporal variations of natural, anthropogenous and social processes (Ed. by A. G. Gamburtsev, Scientific world, 1998) 
3. V. I. Vernadsky, Science (1989)

4. V. I. Keilis-Borok, T. L. Kronrod, G. M. Molchan, Science 82-98, (1982)

5. The change of the geological environment under the influence of human activity (Nedra, 1978)

6. V. A. Minaev, B. N. Trubnikov, V. S. Chudnovsky, Seismic activity as a possible factor causing abnormal behaviour of biological systems and aggravation of the criminal situation/in the $k N$ (Information technology and computer models in the activities of the internal Affairs bodies, Academy of the interior Ministry, 1996)

7. Atlas of temporal variations of natural processes (Ed. by A. G. Gamburtsev, Order and chaos in the lithosphere and other spheres, Publishing House of OIFZ RAN, 1994)

8. Atlas of temporal variations of natural, anthropogenous and social processes (Ed. by A. G. Gamburtsev, Natural and social spheres as part of the environment and as objects of impacts, Moscow, Yanus-K, 2002)

9. I. V. Ananyin, On the change in the electric field intensity before and during the earthquake (In sat: Problems of Seismicity of the East European Platform, Ed. OIFZ RAN, 2000)

10. V. V. Ivanov, B. G. Tarasov, E. D. Kuzmenko, N. Gordiychuk, Geology and Exploration 3, 101-104 (1991)

11. V. A. Saraev, N. T. Ivanova, Geology, Stratigraphy and Mineral Resources of Siberia, 45-48 (1979)

12. V. E. Manilov, Energoatomizdat (1983)

13. I. V. Ananyin, Federal System of Seismological Observations and Prediction of Earthquakes 4, 45-48 (1994)

14. V. A. Minaev, A. O. Faddeev, Geo-ecological risk assessment. Modeling of safety of tourist and recreational areas (Finance and statistics, Publishing House INFRA-M, 2009)

15. O. O. Faddeev, To the question of assessment of zones of geoecological risk in urban and industrial areas (Proceedings of the IV Russian-Ukrainian scientific and technical Symposium "Modern information technologies in science, production and education", Penza, 2004)

16. I. V. Anan'in, Science (1977)

17. Aviation medicine (Ed. by A. N. Babeichuk, Publishing House of DOSAAF of the USSR, 1980)

18. V. A. Minaev, A. O. Faddeev, "Slow" catastrophes, health and safety of the population. XXV scientific and technical conference "security Systems" (Academy of state fire service of EMERCOM of the Russian Federation, 2006)

19. V. A. Minaev, A. O. Faddeev, Landslides, subsidence, karst phenomena as manifestations of "slow" disasters (Proceedings of the XXV scientific-technical conference "safety Systems", Academy of state fire service of EMERCOM of the Russian Federation, 2006)

20. V. A. Minaev, A. O. Faddeev, The Problem of "slow" disasters (Proceedings of the XXV scientific-technical conference "safety Systems", Academy of state fire service of EMERCOM of the Russian Federation, 2006)

21. V. A. Minaev, A. O. Faddeev, Manifestations of "slow" accidents in recreational areas (Southern Urals as the unity of Europe and Asia. Tourism: peace and sustainable development, Proceedings of the international forum, 1, 37-51, Magnitogorsk, 2006) 
22. V. A. Minaev, A. O. Faddeev, R. Danilov, Problems of Risk Management in Technosphere 2, 15, 36-50 (2010)

23. Moscow: Geology and city (Ed. V. I. Osipov, O. P. Medvedev, Moscow textbooks and kartolitografiya, 1997)

24. A. O. Faddeev, Security Management 4, 25-27 (2004)

25. Bioindication of terrestrial ecosystems (Ed. by R. Schubert, Mir, 1988)

26. Dubrov, The Ecology of the home and human health (Ufa, Slovo, 1995)

27. Faddeev, A. O. Geoecological aspect of the functioning of the subdivisions of the UIS and their management. Ryazan: Academy of law and administration of the Ministry of justice of Russia, 2003. 190 p.

28. M. P. Glasco, E. Y. Rantsman, Reports of the Academy of Sciences 350, 3, $397-400$ (1996)

29. U. G. Pirumov, Numerical methods (Moscow, Drofa, 2003)

30. N. F. Zamesov, I. I. Dzema, Forecasting of initial stress fields in ore deposits (Publishing house-IPKON an SSSR, 1987)

31. V. M. Pizengolts, Development of the food sector in crisis (Proceedings of the international scientific conference of the faculty, staff and graduate students of the Russian University, 2009) 